\title{
General decay of solutions for a nonlinear viscoelastic wave equation with nonlocal boundary damping
}

\author{
Faramarz Tahamtani and Amir Peyravi
}




\title{
GENERAL DECAY OF SOLUTIONS FOR A NONLINEAR VISCOELASTIC WAVE EQUATION WITH NONLOCAL BOUNDARY DAMPING
}

\author{
FARAMARZ TAHAMTANI AND AMIR PEYRAVI
}

Received 24 September, 2013

\begin{abstract}
In this work we show that under weaker assumptions on the memory kernel $g$, exponential and polynomial decay rates of the solution energy in Li and Zhao [8] are only special cases. Our result improves earlier results in the literature.
\end{abstract}

2010 Mathematics Subject Classification: 35B40; 35L20; 35L70

Keywords: general decay, boundary damping, viscoelastic

\section{INTRODUCTION}

In this article, we investigate the following initial value problem

$$
\left\{\begin{array}{l}
u_{t t}-k_{0} \Delta u+\int_{0}^{t} g(t-s) \operatorname{div}[a(x) \nabla u(s)] d s+b(x) h\left(u_{t}\right)=0, \\
\quad(x, t) \in \Omega \times(0, \infty), \\
-k_{0} \frac{\partial u}{\partial v}+\int_{0}^{t} g(t-s)(a(x) \nabla u(s)) . v d s=f(u), \quad(x, t) \in \Gamma_{1} \times(0, \infty), \\
u(x, t)=0, \quad(x, t) \in \Gamma_{0} \times(0, \infty), \\
u(x, 0)=u_{0}(x) \quad u_{t}(x, 0)=u_{1}(x), \quad x \in \Omega
\end{array}\right.
$$

where $k_{0}>0, \Omega$ is a bounded domain in $R^{n}(n \geq 1)$ with a smooth boundary $\partial \Omega=$ $\Gamma_{0} \cup \Gamma_{1}, \bar{\Gamma}_{0} \cap \bar{\Gamma}_{1}=\varnothing, \Gamma_{0}$ and $\Gamma_{1}$ are closed with positive measures, $v$ is the unit outward normal to $\partial \Omega, g$ denotes the memory kernel and $a, b, h$ and $f$ are real valued functions which satisfy appropriate conditions.

This problem arises in the study of motion of viscoelastic materials. We refer to $[7,18]$ for mathematical analysis on the motions of materials with memory. The above problem with dirichlet boundary conditions has been considered by many authors. In this regard we recall the pioneer works by Cavalcanti et al. [1,3], Santos [19] and Rivera et al. [15, 16]. 
In [8], Li and Zhao studied the problem (1.1) and proved exponential and polynomial decay results under weaker assumptions on $g$ which improved [17]. In fact, in [17], the authors studied problem (1.1) with nonlinear boundary damping when $f(u)=|u|^{\gamma} u$ and $b(x)=1$ on $\Omega$. Assuming that the kernel $g$ in the memory term decays exponentially, they showed exponential energy decay by using the perturbed energy method provided that $\|g\|_{L^{1}[0, \infty)}$ is small enough. In [9] Li et al. considered a related problem with nonlinear boundary dissipation. Under suitable conditions on the initial data and relaxation function, they established existence and uniqueness of global solutions by means of Galerkin method and showed that the energy decays exponentially if the decay rate of the memory kernel is also exponentially. These results have been recently improved by $\mathrm{Wu}$ and Chen [21] where the authors considered a nonlinear wave equation with boundary dissipation in presence of a local damping term, $b(x) u_{t}$, in $\Omega$. The authors used Lyapunov functions to establish general decay rate of solution energy which is not necessarily of exponential or polynomial type. However, in order to prove main results, they supposed that the function $a(x)$ satisfies

$$
|\nabla a(x)|^{2} \leq \alpha_{1}^{2}|a(x)| .
$$

For more related results about the boundary stabilization we refer to Cavalcanti et al.[2], Liu and Yu [10], Lu et al. [11], Messaoudi and Soufyane [13]. We can also recall some other pioneer papers in connecting with the viscoelasticity such as Sobrinho and Rivera [20], Fabrizio and Polidro [6], Rivera et al. [14] and Dafermos [4].

In this work, we study problem (1.1). We show that for a certain class of relaxation functions, the decay rate of the energy is similar to that of $g$. Therefore, our result improves earlier results in $[8,9]$ where only the exponential and polynomial decay rates are obtained. The main point of the contribution is based on an inequality given by Martinez [12]. In this way, we are allowed to weaken some technical assumptions for the kernel $g$ or even for the function $a(x)$ (such as inequality (1.2) which has been considered in [21]).

\section{PRELIMINARIES}

In this section we present some materials that will be needed throughout the paper. We begin by presenting the precise hypotheses on the problem (1.1).

$\left(\mathrm{H}_{1}\right) a, b: \Omega \rightarrow R$ are positive functions so that $a, b \in L^{\infty}(\Omega)$ and

$$
b(x) \geq b_{0}>0 .
$$

$\left(\mathrm{H}_{2}\right) f: R \rightarrow R$ satisfies

$$
f(s) s \geq 2 F(s) \geq 0, \quad \forall s \in R,
$$

where

$$
F(z)=\int_{0}^{z} f(s) d s .
$$


$\left(\mathrm{H}_{3}\right) h: R \rightarrow R$ is a nondecreasing function, such that for some positive constants $\alpha$ and $\beta$, satisfies

$$
h(s) s \geq \alpha|s|^{2}, \quad|h(s)| \leq \beta|s|, \quad \forall s \in R .
$$

$\left(\mathrm{H}_{4}\right) g:[0, \infty) \rightarrow[0, \infty)$ is a non-increasing $C^{1}$ function such that

$$
g(0)>0, \quad k_{0}-\|a\|_{\infty} \int_{0}^{+\infty} g(s) d s=l>0,
$$

and there exists a non-increasing positive differentiable function $\xi$ such that

$$
g^{\prime}(t) \leq-\xi(t) g(t), \quad \forall t \geq 0, \quad \int_{0}^{+\infty} \xi(s) d s=\infty .
$$

We will also consider the Hilbert space

$$
H_{\Gamma_{0}}^{1}(\Omega)=\left\{u \in H^{1}(\Omega): u=0 \text { on } \Gamma_{0}\right\} .
$$

Lemma 1 (Poincaré inequality). There exists a positive constant $B$ such that

$$
\|u\|_{L^{2}(\Omega)} \leq B\|\nabla u\|_{L^{2}(\Omega)},
$$

for all $u \in H_{\Gamma_{0}}^{1}(\Omega)$.

Referring to [5,17], we state the following existence and uniqueness theorem.

Theorem 1. If $\left(u_{0}, u_{1}\right) \in\left(H^{2}(\Omega) \cap H_{\Gamma_{0}}^{1}(\Omega)\right) \times H_{\Gamma_{0}}^{1}(\Omega)$, then the problem (1.1) has a unique solution satisfying

$$
\begin{gathered}
u \in L_{l o c}^{\infty}\left(0, \infty ; H_{\Gamma_{0}}^{1}(\Omega) \cap H^{2}(\Omega)\right), \quad u_{t} \in L_{l o c}^{\infty}\left(0, \infty ; H_{\Gamma_{0}}^{1}(\Omega)\right), \\
u_{t t} \in L_{l o c}^{\infty}\left(0, \infty ; L^{2}(\Omega)\right) .
\end{gathered}
$$

Moreover

$$
u \in C\left([0, \infty) ; H_{0}^{1}(\Omega)\right), \quad u_{t} \in C\left([0, \infty) ; L^{2}(\Omega)\right) .
$$

Finally, we present the following lemma by Martinez [12] which plays important role in our proof.

Lemma 2. Let $E: \mathbb{R}_{+} \rightarrow \mathbb{R}_{+}$be a nonincreasing function and $\psi: \mathbb{R}_{+} \rightarrow \mathbb{R}_{+}$be a $C^{2}$ increasing function such that $\psi(0)=0$ and $\lim _{t \rightarrow+\infty} \psi(t)=+\infty$. Assume that there exists $c>0$ for which

$$
\int_{t}^{+\infty} \psi^{\prime}(s) E(s) d s \leq c E(t), \quad \forall t \geq 0,
$$

then

$$
E(t) \leq \lambda E(0) e^{-\omega \psi(t)},
$$

for some positive constants $\omega$ and $\lambda$. 


\section{ENERGY DECAY}

In this section we state and prove our main result. First, we define the energy related to problem (1.1) as in [8]

$$
\begin{gathered}
E(t)=\frac{1}{2} \int_{\Omega}\left|u_{t}(t)\right|^{2} d x+\frac{1}{2} \int_{\Omega}\left(k_{0}-a(x) \int_{0}^{t} g(s) d s\right)|\nabla u(t)|^{2} d x \\
+\frac{1}{2}(g \circ \nabla u)(t)+\int_{\Gamma_{1}} F(u) d \Gamma,
\end{gathered}
$$

where

We also observe that

$$
(g \circ \nabla u)(t)=\int_{\Omega} \int_{0}^{t} g(t-s) a(x)|\nabla u(t)-\nabla u(s)|^{2} d s d x .
$$

$$
\begin{gathered}
\int_{\Omega}\left(k_{0}-a(x) \int_{0}^{t} g(s) d s\right)|\nabla u(t)|^{2} d x \geq \\
\left(k_{0}-\|a\|_{\infty} \int_{0}^{t} g(t) d t\right)\|\nabla u(t)\|_{2}^{2} \geq l\|\nabla u(t)\|_{2}^{2} .
\end{gathered}
$$

Lemma 3 (Lemma 2.3 of [8]). The energy function $E(t)$ satisfies $E(t) \geq 0$ and

$$
E^{\prime}(t)=\frac{1}{2}\left(g^{\prime} \circ \nabla u\right)(t)-\frac{1}{2} g(t) \int_{\Omega} a(x)|\nabla u(t)|^{2} d x-\int_{\Omega} b(x) h\left(u_{t}\right) u_{t} d x \leq 0 .
$$

Theorem 2. Assume that $\left(u_{0}, u_{1}\right) \in\left(H^{2}(\Omega) \cap H_{\Gamma_{0}}^{1}(\Omega)\right) \times H_{\Gamma_{0}}^{1}(\Omega),\left(H_{1}\right)-\left(H_{3}\right)$ and (2.5) hold, and

(i) If

$$
a(x) \geq a_{0}>0 .
$$

$$
g^{\prime}(t) \leq-c g(t), c>0,
$$

then, the energy $E(t)$ of problem (1.1) satisfies the decay rate

$$
E(t) \leq 4 E(0) e^{-C t}, \quad \forall t \geq T .
$$

(ii) If

$$
g^{\prime}(t) \leq-c g^{1+\frac{1}{p}}(t), \quad p>2, c>0,
$$

then, the energy $E(t)$ of problem (1.1) satisfies the decay rate

for some $C, T>0$.

$$
E(t) \leq \frac{C}{(1+t)^{p}}, \quad \forall t \geq T,
$$

Proof. See [8], Theorems 3.1 and 3.2.

In the next theorem, we state our main result. We extend the above rates of decay to a more general case which is similar to that of $g$. In fact, we use the assumption (2.6) which is weaker than (3.4) and (3.5) . 
Theorem 3. Assume that $\left(\mathrm{H}_{1}\right)-\left(\mathrm{H}_{4}\right)$ hold. If the initial data $\left(u_{0}, u_{1}\right) \in\left(H^{2}(\Omega) \cap\right.$ $\left.H_{\Gamma_{0}}^{1}(\Omega)\right) \times H_{\Gamma_{0}}^{1}(\Omega)$, then the solution of problem (1.1) satisfies

$$
E(t) \leq K E(0) e^{-\kappa \int_{0}^{t} \xi(s) d s},
$$

for some $K, \kappa>0$.

Proof. Multiplying $(1.1)_{1}$ by $\xi(t) u$ and integrating over $\Omega \times\left[t_{1}, t_{2}\right]\left(0 \leq t_{1} \leq t_{2}\right)$, we get

$$
\begin{gathered}
\int_{t_{1}}^{t_{2}} \xi(t) \int_{\Omega} u u_{t} d x d t+k_{0} \int_{t_{1}}^{t_{2}} \xi(t) \int_{\Omega}|\nabla u(t)|^{2} d x d t \\
+\int_{t_{1}}^{t_{2}} \xi(t) \int_{\Omega} b(x) u h\left(u_{t}\right) d x d t \quad+\int_{t_{1}}^{t_{2}} \xi(t) \int_{\Gamma_{1}} u f(u) d \Gamma d t \\
-\int_{t_{1}}^{t_{2}} \xi(t) \int_{\Omega} \nabla u(t) \cdot \int_{0}^{t} g(t-s)(a(x) \nabla u(s)) d s d x d t=0 .
\end{gathered}
$$

For the last term in the right hand side of (3.7) we have

$$
\begin{aligned}
\int_{\Omega} \nabla u(t) \cdot \int_{0}^{t} g(t-s)(a(x) \nabla u(s)) d s d x \\
=\int_{\Omega} \nabla u(t) \cdot \int_{0}^{t} g(t-s) a(x)(\nabla u(s)-\nabla u(t)) d s d x \\
\quad+\int_{0}^{t} g(s) d s \int_{\Omega} a(x)|\nabla u(t)|^{2} d x .
\end{aligned}
$$

Substituting (3.8) in (3.7) and using (2.2), (2.3) and (3.1) we obtain

$$
\begin{gathered}
2 \int_{t_{1}}^{t_{2}} \xi(t) E(t) d t \\
\leq-\int_{t_{1}}^{t_{2}} \xi(t) \int_{\Omega} u u_{t} d x d t+\int_{t_{1}}^{t_{2}} \xi(t)\left\|u_{t}\right\|_{2}^{2} d t-\int_{t_{1}}^{t_{2}} \xi(t) \int_{\Omega} b(x) u h\left(u_{t}\right) d x d t \\
+\int_{t_{1}}^{t_{2}} \xi(t) \int_{\Omega} \nabla u(t) \cdot \int_{0}^{t} g(t-s) a(x)(\nabla u(s)-\nabla u(t)) d s d x d t \\
+\int_{t_{1}}^{t_{2}} \xi(t)(g \circ \nabla u)(t) d t .
\end{gathered}
$$

For the first term in the right hand side of (3.9) we have

$$
\begin{gathered}
-\int_{t_{1}}^{t_{2}} \xi(t) \int_{\Omega} u u_{t} d x d t \\
=-\left.\int_{\Omega} \xi(t) u u_{t} d x\right|_{t_{1}} ^{t_{2}}+\int_{t_{1}}^{t_{2}} \xi(t) \int_{\Omega} u u_{t} d x d t+\int_{t_{1}}^{t_{2}} \xi(t)\left\|u_{t}\right\|_{2}^{2} d t .
\end{gathered}
$$


By Lemma 1 and (3.1) we have

$$
\begin{aligned}
& \left.\left|-\int_{\Omega} \xi(t) u u_{t} d x\right|_{t_{1}}^{t_{2}}\left|\leq \sum_{i=1}^{2}\right| \xi(t) \int_{\Omega} u u_{t} d x\right|_{t=t_{i}} \\
& \leq \sum_{i=1}^{2}\left[\xi(t)\left(\frac{B^{2}}{2}\|\nabla u\|_{2}^{2}+\frac{1}{2}\left\|u_{t}\right\|_{2}^{2}\right)\right]_{t=t_{i}} \\
& \leq \sum_{i=1}^{2}\left[\left(\frac{B^{2}}{l}+1\right) \xi(t) E(t)\right]_{t=t_{i}} \leq 2\left(\frac{B^{2}+l}{l}\right) \xi\left(t_{1}\right) E\left(t_{1}\right) .
\end{aligned}
$$

Similarly,

$$
\begin{aligned}
\left|\int_{t_{1}}^{t_{2}} \xi^{\prime}(t) \int_{\Omega} u u_{t} d x d t\right| & \leq \int_{t_{1}}^{t_{2}}\left|\xi^{\prime}(t)\right|\left(\frac{B^{2}}{2}\|\nabla u\|_{2}^{2}+\frac{1}{2}\left\|u_{t}\right\|_{2}^{2}\right) \\
\leq & -\left(\frac{B^{2}+l}{l}\right) \int_{t_{1}}^{t_{2}} \xi^{\prime}(t) E(t) d t \leq\left(\frac{B^{2}+l}{l}\right) \xi\left(t_{1}\right) E\left(t_{1}\right) .
\end{aligned}
$$

To estimate the last term in the right-hand side of (3.10), we use (3.2), (2.1) and (2.4) to obtain

$$
E^{\prime}(t) \leq-\int_{\Omega} b(x) h\left(u_{t}\right) u_{t} d x \leq-b_{0} \int_{\Omega} h\left(u_{t}\right) u_{t} d x \leq-b_{0} \alpha\left\|u_{t}\right\|_{2}^{2} .
$$

Therefore,

$$
\int_{t_{1}}^{t_{2}} \xi(t)\left\|u_{t}\right\|_{2}^{2} d t \leq-\frac{1}{b_{0} \alpha} \int_{t_{1}}^{t_{2}} \xi(t) E^{\prime}(t) d t \leq \frac{1}{b_{0} \alpha} \xi\left(t_{1}\right) E\left(t_{1}\right) .
$$

From (3.10)-(3.13) we get

$$
\left|-\int_{t_{1}}^{t_{2}} \xi(t) \int_{\Omega} u u_{t t} d x d t\right|+\int_{t_{1}}^{t_{2}} \xi(t)\left\|u_{t}\right\|_{2}^{2} d t \leq\left(3 l^{-1}\left(B^{2}+l\right)+\frac{2}{b_{0} \alpha}\right) \xi\left(t_{1}\right) E\left(t_{1}\right) .
$$

By (2.4), Young's inequality, (3.1) and (3.2) we arrive at

$$
\begin{gathered}
\left|-\int_{t_{1}}^{t_{2}} \xi(t) \int_{\Omega} b(x) u h\left(u_{t}\right) d x d t\right| \leq \beta \int_{t_{1}}^{t_{2}} \xi(t) \int_{\Omega} b(x)\left(\frac{\delta B^{2}}{2}|\nabla u|^{2}+\frac{1}{2 \delta}\left|u_{t}\right|^{2}\right) d x d t \\
\leq \frac{\delta}{2} \beta B^{2}\|b\|_{\infty} \int_{t_{1}}^{t_{2}} \xi(t)\|\nabla u\|_{2}^{2} d t-\frac{\beta}{2 \delta \alpha} \int_{t_{1}}^{t_{2}} \xi(t) E^{\prime}(t) d t \\
\leq \frac{\delta}{l} \beta B^{2}\|b\|_{\infty} \int_{t_{1}}^{t_{2}} \xi(t) E(t) d t+\frac{\beta}{2 \delta \alpha} \xi\left(t_{1}\right) E\left(t_{1}\right) .
\end{gathered}
$$

Also, we have

$$
\int_{\Omega} a(x) \nabla u(t) \cdot \int_{0}^{t} g(t-s)(\nabla u(s)-\nabla u(t)) d s d x
$$




$$
\begin{aligned}
& \leq \delta \int_{\Omega} a(x)|\nabla u(t)|^{2} d x+\frac{1}{4 \delta} \int_{\Omega} a(x)\left(\int_{0}^{t} g(t-s)(\nabla u(s)-\nabla u(t)) d s\right)^{2} d x \\
& \leq \delta\|a\|_{\infty}\|\nabla u\|_{2}^{2}+\frac{1}{4 \delta} \int_{0}^{t} g(s) d s \int_{\Omega} \int_{0}^{t} g(t-s) a(x)|\nabla u(s)-\nabla u(t)|^{2} d s d x \\
& \leq \frac{2 \delta}{l}\|a\|_{\infty} E(t)+\frac{k_{0}-l}{4 \delta \|_{\infty}}(g \circ \nabla u)(t) .
\end{aligned}
$$

By (2.6) we have

$$
\xi(t)(g \circ \nabla u)(t) \leq-\left(g^{\prime} \circ \nabla u\right)(t) \leq-2 E^{\prime}(t) .
$$

Finally, using (3.14)-(3.17), the estimate (3.9) takes the form

$$
\begin{aligned}
& {\left[2-\frac{\delta}{l}\left(\beta B^{2}\|b\|_{\infty}+2\|a\|_{\infty}\right)\right] \int_{t_{1}}^{t_{2}} \xi(t) E(t) d t} \\
& \leq\left(3 l^{-1}\left(B^{2}+l\right)+\frac{2}{b_{0} \alpha}+\frac{\beta}{2 \delta \alpha}\right) \xi\left(t_{1}\right) E\left(t_{1}\right)-\left(\frac{k_{0}-l}{2 \delta\|a\|_{\infty}}+2\right) \int_{t_{1}}^{t_{2}} E^{\prime}(t) d t \\
& \leq\left[\left(3 l^{-1}\left(B^{2}+l\right)+\frac{2}{b_{0} \alpha}+\frac{\beta}{2 \delta \alpha}\right) \xi(0)+\frac{k_{0}-l}{2 \delta\left\|_{0}\right\|_{\infty}}+2\right] E\left(t_{1}\right)
\end{aligned}
$$

Choosing $\delta$ small enough and letting $t_{2}$ go to infinity, we rewrite (3.18) as

$$
\int_{t}^{+\infty} \xi(t) E(t) d t \leq \lambda E(t), \quad \forall t \geq 0,
$$

for some $\lambda>0$. Then, the assumptions of Lemma 2 satisfied with $\psi(t)=\int_{0}^{t} \xi(s) d s$. Therefore (3.6) is established and the proof of Theorem 3 is now complete.

Remark 1. We note that similar rates of decay were given in [21]. However, we did not use (3.3) which has been supposed in [8]- $\mathrm{H}_{1}$ and [21]- $\mathrm{A}_{3}$.

\section{REFERENCES}

[1] M. M. Cavalcanti, V. N. Domingos Cavalcanti, T. F. Ma, and J. A. Soriano, "Global existence and asymptotic stability for viscoelastic problems," Differential Integral Equations, vol. 15, pp. 731-748, 2002.

[2] M. M. Cavalcanti, V. N. Domingos Cavalcanti, and P. Martinez, "General decay rate estimates for viscoelastic dissipative systems," Nonlinear Anal, vol. 68, pp. 177-193, 2008.

[3] M. M. Cavalcanti, V. N. Domingos Cavalcanti, J. Prates Filho, and J. Soriano, "Existence and uniform decay rates for viscoelastic problems with nonlinear boundary damping," Differential Integral Equations, vol. 14, pp. 85-116, 2001.

[4] C. M. Dafermos, "Asymptotic stability in viscoelasticity," Arch. Ration. Mech. Anal, vol. 36, pp. 297-308, 1970.

[5] L. C. Evans, Partial differential equations., ser. Graduate Studies in Mathematics. Providence, RI: American Mathematical Society, 1998, vol. 19.

[6] M. Fabrizio and S. Polidoro, "Asymptotic decay for some differential systems with fading memory," Appl. Anal, vol. 81, no. 6, pp. 1245-1264, 2002. 
[7] M. Fabrizio and A. Morro, Mathematical problems in linear viscoelasticity, ser. SIAM Studies in Applied Mathematics. Philadelphia: SIAM, 1992, vol. 12.

[8] F. Li and C. Zhao, "Uniform decay rates for nonlinear viscoelastic wave equation with nonlocal boundary damping," Nonlinear Anal, vol. 74, pp. 3468-3477, 2011.

[9] F. Li, C. Zhao, and Y. Chen, "Global existence, uniqeness and decay estimates for nonlinear viscoelastic wave equation with boundary dissipation," Nonlinear Anal. Real World Appl, vol. 12, pp. 1759-1773, 2011.

[10] W. J. Liu and J. Yu, "On decay and blow-up of the solution for a viscoelastic wave equation with boundary damping and source terms," Nonlinear Anal, vol. 74, no. 6, pp. 2175-2190, 2011.

[11] L. Lu, S. Li, and S. Chai, "On a viscoelastic equation with nonlinear boundary damping and source terms: global existence and decay of the solution," Nonlinear Anal. Real World Appl, vol. 12, no. 1, pp. 295-303, 2011.

[12] P. Martinez, "A new method to obtain decay rate estimates for dissipative systems," ESAIM Control Optim. Calc. Var, vol. 4, no. 1, pp. 419-444, 1999.

[13] S. A. Messaoudi and A. Soufyane, "General decay of solutions of a wave equation with a boundary control of memory type," Nonlinear Anal. Real World Appl, vol. 11, no. 4, pp. 2896-2904, 2010.

[14] J. E. Munoz Rivera, E. Lapa, and R. Barreto, "Decay rates for viscoelastic plates with memory," J. Elasticity, vol. 64, pp. 61-87, 1996.

[15] J. E. Munoz Rivera and A. Peres Salvatierra, "Asymptotic behaviour of the energy in partially viscoelastic materials," Quart. Appl. Math, vol. 59, pp. 557-578, 2001.

[16] J. E. Munoz Rivera and J. B. Sobrinho, "Existence and uniform rates of decay for contact problems in viscoelasticity,” Appl. Anal, vol. 67, pp. 175-199, 1997.

[17] J. Y. Park and S. H. Park Busan, "Existence and asymptotic stability for viscoelastic problems with nonlocal boundary dissipation," Czechoslovak Math. J, vol. 56, no. 131, pp. 273-286, 2006.

[18] M. Renardy, W. J. Hrusa, and J. A. Nohel, Mathematical Problems in Viscoelasticity, ser. Pitman Monographs and Surveys in Pure and Applied Mathematics. New York: John Wiely and Sons, 1987, vol. 35.

[19] M. Santos, "Decay rates for solutions of a system of wave equations with memory," Electron. $J$. Differential Equations, vol. 44, pp. 1-17, 2002.

[20] J. B. Sobrinho and J. E. Munoz Rivera, "Existence and uniform rates of decay for contact problems in viscoelasticity," App. Anal, vol. 67, pp. 3-4, 1997.

[21] S. T. Wu and H. F. Chen, "Uniform decay of solutions for a nonlinear viscoelastic wave equation with boundary dissipation,” J. Funct. Spaces Appl, no. 10.1155/2012/421847, 2012, 2012.

Authors' addresses

Faramarz Tahamtani

Department of Mathematics, College of Sciences, Shiraz University, Shiraz, 71457-44776, Iran

E-mail address: tahamtanieshirazu.ac.ir

Amir Peyravi

Department of Mathematics, College of Sciences, Shiraz University, Shiraz, 71457-44776, Iran

E-mail address: peyravi@shirazu.ac.ir 